\title{
Funções de autocorrelação em sistemas fluidos longe do equilíbrio: um protótipo do fenômeno de autocorrelação em sistemas naturais
}

\author{
Carolina Casagrande Blanco \\ Antônio Gledson de Oliveira Goulart \\ Gervásio Annes Degrazia
}

\section{RESUMO}

A autocorrelação surge como um fenômeno natural presente em sistemas longe da situação de equilíbrio termodinâmico e expressa um comportamento de memória contido nesses estados complexos. Pelo fato de sistemas biológicos serem intrinsecamente mais complexos do que os estados em que se encontram os diferentes sistemas físicos, é mais fácil analisarmos o comportamento de memória associado a estes sistemas. Isto envolve formas matemáticas mais simples que apresentam soluções diretas e facilmente analisáveis. Neste estudo, utiliza-se o Teorema de Wiener-Kintchin na derivação de formas de funções de correlação associadas a fluxos turbulentos, que por sua vez, são sistemas físicos dissipativos longe do equilíbrio e que apresentam características inerentes a sistemas biológicos. 


\section{SUMMARY}

The autocorrelation rises like natural phenomena in farfrom-equilibrium thermodynamic systems, providing a memory behavior for these complex states. For the fact that biological systems are in an intrinsically more complex state than that of the different physical ones, it becomes easier for us to analyze the memory behavior showed by this kind of system. It involves more simple mathematical methods, which have direct, and easily solutions for analysis. In this paper, Wiener-Kintchin's Theorem is used to derive forms of correlation functions associated to turbulent fluxes, kinds of dissipative far-fromequilibrium physical systems, which display inherent biological systems characteristics.

\section{INTRODUÇÃO}

A Segunda Lei da Termodinâmica afirma que sistemas equilibrados, quando 'perturbados' por vínculos externos de energia, sempre tendem a restabelecer o equilíbrio. Neste estado, o sistema alcança a entropia máxima, onde a homogeneidade entre as suas partes prevalece. No entanto, quando essas perturbações alcançam um determinado nível crítico, o sistema se aproxima dos pontos de bifurcação; as flutuações de estado são intensificadas e surgem correlações macroscópicas entre suas partes. É neste ponto preciso de transição do equilíbrio ao não-equilíbrio que os eventos locais repercutem ao longo de todo o sistema (PRIGOGINE, I., SENGERS, I., 1984).

Num ponto de bifurcação, somente a chance determinará a evolução posterior do sistema. O fato é que, entre muitas escolhas, a que foi mantida conferirá ao sistema uma dimensão histórica; algum tipo de 'memória' de um evento passado que se estabeleceu num momento crítico. Quanto mais um sistema é afastado do equilíbrio, mais sofisticados são seus mecanismos para resistir ao afastamento. Se as condições dinâmicas e/ou cinéticas permitirem, surgirão processos de auto-organização que promovem a dissipação de gradientes impostos pelo vínculo ao sistema. É o nascimento da ordem a partir da desordem na forma de estruturas dissipativas complexas através da auto-organização, e essa complexidade parece ser uma conseqüência inevitável aos sistemas longe do equilíbrio quando condições adequadas são satisfeitas (NICOLIS, 1989).

Neste sentido, a Segunda Lei da Termodinâmica 
promove a auto-organização e determina a direção de muitos dos processos observados no desenvolvimento dos sistemas complexos. Logo, esta lei também pode ser enunciada como a medida quantitativa da irreversibilidade - a entropia. Qualquer processo real, enquanto sistema aberto, somente pode prosseguir em uma direção que resulte em aumento de entropia. Porém, aqui, a entropia máxima (equilíbrio termodinâmico) significa desagregação e morte. Sistemas organizados não vivos e sístemas vivos dependem de fluxos energéticos externos para manter sua organização e dissipam gradientes de energia para realizar esses processos de auto-organização. Tal organização é mantida à custa de diminuir a entropia do sistema (KAY, J., SCHNEIDER, E., 1992).

Podemos considerar um fluxo turbulento como um sistema físico dissipativo longe da condição de equilíbrio termodinâmico e composto por uma superposição de turbilhões.

Uma interação contínua entre estes turbilhões é condicionada pelo fenômeno de autocorrelação, e este grau de organização como um todo é mantido pela interação do sistema com o mecanismo de forçante, do qual ele extrai a sua energia. Uma questão interessante é conhecer como a energia cinética distribuise de acordo com as várias escalas do movimento turbulento.

O estudo de tal complexidade em sistemas físicos oferece um protótipo do comportamento dos sistemas biológicos, onde a complexidade é inerente à condição de sistemas vivos.

\section{METODOLOGIA}

Neste estudo, utiliza-se o Teorema de Wiener-Kintchin na derivação de formas de funções de correlação associadas a fluxos turbulentos.

O Teorema de Wiener-Kintchin (1) relaciona, através da Transformada de Fourier, o espectro de energia com a função de autocorrelação. Partindo-se de diferentes formas da função espectro de energia turbulenta, pode-se derivar diferentes expressões de autocorrelação.

$$
R_{L}(t)=\int_{0}^{\infty} S_{L}(n) \cos (2 \pi n t) d n
$$


Seguindo SORBJAn (1989), um espectro mede a distribuição da variância de uma variável nas diferentes freqüências ou comprimentos de ondas. Se a variável é uma componente da velocidade turbulenta de uma partícula do fluido, o espectro descreve a distribuição da energia cinética nas diferentes freqüências. O Teorema de Wiener-Kintchin estabelece um resultado fundamental que relaciona a Transformada de Fourier do espectro de energia, à função de autocorrelação.

Neste trabalho, como forma espectral, foi considerada a função para o espectro Lagrangeano de energia turbulenta:

$$
S_{L}(n)=\frac{4 t_{L}}{\left(1+4 n t_{L}\right)^{2}}
$$

A substituição deste espectro no Teorema de WienerKintchin permite obter a seguinte função de correlação:

$$
R_{L}(t)=4 t_{L} \int_{0}^{\infty} \frac{\cos 2 \pi n t}{\left(1+4 n t_{L}\right)^{2}} d n
$$

A solução desta integral é bastante complexa, mas é expressa em termos de uma formulação analítica (4) (ACHESON, 1997), ou seja, todas as funções que aparecem na solução podem ser analisadas e investigadas em detalhes:

$$
R_{L}(t)=-\frac{1}{32 t_{L}{ }^{2}}\left\{2 \pi^{2} t \cos \left(\frac{\pi t}{2 t_{L}}\right)+4 \pi t \operatorname{sen}\left(\frac{\pi t}{2 t_{L}}\right) C_{i}\left(\frac{\pi t}{2 t_{L}}\right)-4 \pi \cos \left(\frac{\pi t}{2 t_{L}}\right) S_{i}\left(\frac{\pi t}{2 t_{L}}\right)-8 t_{L}\right\}
$$

Analisando, posteriormente, o gráfico da função de correlação (5), pode-se vislumbrar a solução da integral (3), expressa pela fórmula complexa (4): 


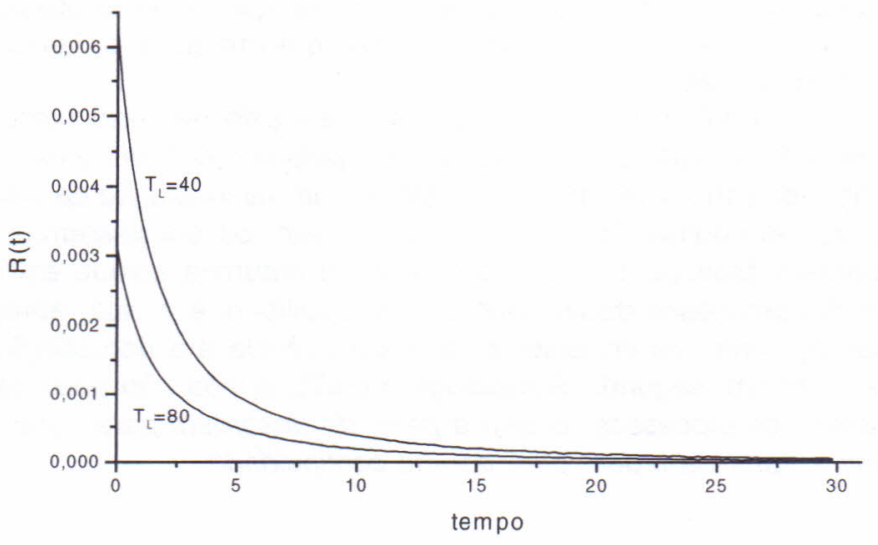

(5)

\section{RESULTADOS E DISCUSSÃO}

A solução da função de correlação (5), como expressa pela formulação analítica obtida, mostra que essa função decai no tempo, ou seja, o nosso sistema dinâmico (no caso o campo turbulento) perde memória ao longo do tempo, descaracterizando-se o fenômeno de autocorrelação. É justamente nesse processo de decaimento da correlação que reside a distinção fundamental entre o comportamento de sistemas organizados não-vivos e sistemas vivos (DAVIS, 1980).

No sistema físico estudado neste trabalho, o decaimento da autocorrelação inevitavelmente leva o sistema a sucumbir. Contudo, considerando sistemas vivos como sistemas dinâmicos dissipativos com memórias codificadas (os genes), tal decaimento não é permitido, e as entidades dissipativas continuam, evidenciando o nascimento da ordem a partir da ordem.

Seja qual for o tipo de sistema organizado, para que ele se mantenha em um estado constante de não-equilíbrio, a troca de entropia precisa ser negativa e igual à entropia gerada por processos internos (ROSS, J., VIDAL, C., 1988). Em sistemas vivos, tais pro- 
cessos internos são ditos metabolismo. O gradiente de energia do sol impulsiona o processo de vida. A luta generalizada pela existência por parte de seres animados é a luta pela entropia, que se torna disponível através da transição de energia do sol quente para a Terra fria (BOLTZMANN, 1886).

KAY (1984) sugere que a vida existe na Terra como um outro meio de dissipar o gradiente imposto pelo sol (reduzindo os gradientes de radiação na Terra) e, como tal, é uma manifestação da Segunda Lei da Termodinâmica, reformulada. Assim, os ecossistemas são componentes bióticos, físicos e químicos da natureza agindo em conjunto como processos dissipativos de não-equilíbrio, e a vida representa o balanço entre os imperativos da sobrevivência e a degradação de energia. Por fim, segundo Schrödinger (1997), a vida não pode existir sem ambos os processos, ordem a partir da desordem para gerar vida e ordem a partir da ordem para dar-Ihe continuidade.

\section{CONCLUSÕES}

O fato de que os sistemas biológicos possuem propriedades capazes de reproduzir as partes que o constituem torna incipiente a idéia de que o comportamento de um fluxo turbulento possa ser aplicado, unicamente, para a descrição da evolução de tais sistemas no tempo. Os inúmeros mecanismos que atuam como forçantes de energia para manter a autocorrelação nesses sistemas é uma das propriedades que maximizam a coerência quando comparadas às de um sistema físico. Do mesmo modo, a própria complexidade das partes que constituem um sistema biológico é um fator que torna o seu comportamento de uma magnitude e complexidade até então não exploradas completamente.

Contudo, este trabalho vem justamente relatar o comportamento de um sistema físico, no caso um fluxo turbulento, possibilitando vislumbrar um protótipo desses fenômenos no mundo biológico.

\section{AGRADECIMENTOS}

Trabalho parcialmente financiado pelo Conselho Nacional de Desenvolvimento Científico e Tecnológico (CNPq) e pela Fundação de Amparo a Pesquisa do Rio Grande do Sul (FAPERGS). 


\section{REFERÊNCIAS BIBLIOGRÁFICAS}

ACHESON, D. (1997). From calculus to chaos - An introduction to dynamics. Oxford University Press. New York.

BOLTZMANN, L. The second law of thermodynamics (1886). Reprinted In: MCGUINESS, B. (Ed.) Boltzmann, Theoretical Physics and Philosophical Problems. New York: D. Reidel, 1974.

DAVIS, BERNARD D., (1980). Frontiers of the Biological Sciences. Sci. July, p. 78.

KAY, J. J. Self-Organization in Living Systems. PhD. (Thesis in Systems Design Engineering) - University of Waterloo, Ontario, 1984.

KAY, J. \& SCHNEIDER, E. Thermodynamics and measures of ecosystem integrity. In: MCKENZIE, D., HYATT, D., MCDONALD, J. (Ed.) Ecological Indicators. New York: Elsevier, 1992.

MEINHARDT, H. (1982). Models of biological pattern formation. Academic. London.

NICOLIS, G. (1989). The new physics. New York.

NICOLIS, G. \& PRIGOGINE, I. (1977). Self-organization in nonequilibrium systems. Wiley. New York.

PRIGOGINE, I. \& SENGERS, I., (1984). Order out of chaos - Man's new dialogue with nature. Bantam Books. New York.

ROSS, J., MÜLLER, S. \& VIDAL, C., (1988). Chemical Waves. Science 240,460 .

SORBJAN, S., (1989). Structure of the Atmospheric Boundary Layer. Prentice Hall. New Jersey. 
CAROLINA CASAGRANDE BLANCO ANTÔNIO GLEDSON DE OLIVEIRA GOULART GERVÁSIO ANNES DEGRAZIA Departamento de Física CCNE - UFSM Santa Maria, RS 